\title{
Measurement of the spectral momentum distribution of valence electrons in amorphous carbon by $(e, 2 e)$ spectroscopy
}

\author{
A. S. Kheifets, J. Lower, K. J. Nygaard, S. Utteridge, M. Vos, and E. Weigold* \\ Electronic Structure of Materials Centre, The Flinders University of South Australia, G.P.O. Box 2100, Adelaide 5001, \\ Australia \\ A. L. Ritter \\ Virginia Tech, Blacksburg, Virginia 24061 \\ (Received 6 August 1993)
}

\begin{abstract}
The spectral momentum density of the valence band of arc evaporated amorphous carbon has been measured by $(e, 2 e)$ spectroscopy with significantly improved energy resolution relative to earlier studies. The valence band has been studied over a range of momenta from 0 to 1.6 a.u. with a resolution of 0.15 a.u. and over a range of binding energies from $9 \mathrm{eV}$ above to $35 \mathrm{eV}$ below the Fermi energy with a resolution of $1.5 \mathrm{eV}$. As seen in earlier studies, two major peaks are observed in the spectral momentum density which previously have been associated with $\sigma$ and $\pi$ bands in graphite. A third feature in the spectra, a weak shoulder approximately $4 \mathrm{eV}$ below the Fermi energy, is observed. A heuristic model is introduced based on the assumption that the spectral momentum density of evaporated amorphous carbon is an angular average of the spectral momentum density of graphite. The behavior of the strongest feature in the experimental spectra is described well by this model, but the other two features, which are in the energy range of the graphitic $\pi, \sigma_{2}$, and $\sigma_{3}$ bands, are poorly represented by the model. It is suggested that the poor agreement is due to rehybridization of these graphitic bands.
\end{abstract}

\section{INTRODUCTION}

The nature of amorphous carbon films has been a subject of intense research in the last few years for both intellectual and technological reasons. ${ }^{1,2}$ Amorphous carbon displays a wide spectrum of structural and electronic properties varying according to the preparation conditions from black, soft, conducting graphitic films to hard, transparent, insulating diamondlike carbon films. These disparate properties arise because of the rich variety of possible carbon-carbon bonds from linear $\left(s p^{1}\right)$, seen in some molecular compounds, to trigonal $\left(s p^{2}\right)$ and tetrahedral $\left(s p^{3}\right)$ bonding present in graphite and diamond, respectively. Though it is generally agreed that graphitic films have a preponderance of $s p^{2}$ bonds, much controversy exists over the nature of the local bond in diamondlike carbon. This paper reports the results of an investigation of graphitic amorphous carbon prepared by arc evaporation.

The electronic structure of graphitic amorphous carbon was studied by $(e, 2 e)$ spectroscopy with higher energy resolution than earlier studies.,4 Our results are in general agreement with other work taking into consideration the difference in resolution. With $(e, 2 e)$ spectroscopy, one can measure directly the momentum density of electron states as a function of valence band energy and, in principle, discriminate unambiguously between different forms of bonding. ${ }^{5,6}$ In practice, one is hampered in the interpretation of the measured spectral momentum density by the lack of a first-principles theory for the electronic structure of amorphous solids. We have constructed a heuristic model of the spectral momentum density of graphitic amorphous carbon based on the assumption that it is an angular average of the spectral momentum density of the crystalline graphite. Though this model cannot be applied to the structure of amorphous carbon (the diffraction pattern of amorphous carbon is not an angular average of the graphite diffraction), some important features of the spectral momentum density are reproduced by our model. Other features in the spectra are not in agreement with predictions of the model and a more fundamental theory clearly is necessary.

The remainder of the material in this paper is organized as follows. In Sec. II, the $(e, 2 e)$ technique is discussed, followed by a description of the apparatus (Sec. III). The experimental results are presented in Sec. IV. Our model for the spectral momentum density of graphitic amorphous carbon is described in Sec. V and the predicted spectrum based on this model is compared with experiment in the same section. A review of our conclusions is given in Sec. VI.

\section{METHOD}

\section{A. General}

Consider an electron in a solid with binding energy $\epsilon$ measured with respect to the chemical potential (referred 
to as the Fermi energy in the remainder of this paper) and with real momentum q. In crystalline solids, the binding energy and momentum are correlated through the dispersion relation $\epsilon=E_{j \mathbf{k}}$ where $j$ is the band in$\operatorname{dex}, \mathbf{k}$ is in the first Brillouin zone, and $\mathbf{q}=\mathbf{k}+\mathbf{G}$ with $\mathbf{G}$ being a reciprocal lattice vector. In amorphous solids, there is no a priori reason for binding energy and momentum to be correlated since translational symmetry is not present. The distinctive feature of the $(e, 2 e)$ method is that the spectral momentum density of electrons in crystalline and amorphous solids can be directly determined from conservation of energy and momentum. Let $E_{0}$ and $\mathbf{p}_{0}$ be the energy and momentum of the incident electron and $E_{A, B}$ and $\mathbf{p}_{A, B}$ be the energies and momenta of the scattered and ejected electrons. The binding energy and momentum of the target electron are

$$
\begin{gathered}
\epsilon=E_{A}+E_{B}-E_{0}, \\
\mathbf{q}=\mathbf{p}_{A}+\mathbf{p}_{B}-\mathbf{p}_{0} .
\end{gathered}
$$

The scattered and ejected electrons $(A, B)$ are detected in coincidence to ensure that they originate from the same specific ionization event. The spectral momentum density $\rho(\epsilon, \mathbf{q})$ is then proportional to the net coincidence count rate as a function of $\mathbf{q}$ and $\epsilon$.

The scattering geometry used in this experiment is defined in Fig. 1. The $z$ axis is taken to be in the direction of the incident momentum and the $x$ and $y$ axes are parallel and perpendicular to the collision plane, respectively. Different momenta can be selected in this experiment in three ways. The $y$ component can be varied by tilting the incident beam direction up or down by an angle $\phi / 2$ with respect to the collision plane while keeping $E_{A}=E_{B}$ and $\theta_{A}=\theta_{B}=45^{\circ}$. In the small angle limit

$$
q_{y}=p_{0} \frac{\phi}{2}, \quad q_{x}=q_{z}=0
$$

The $z$ component was varied by symmetrically sweeping the polar angles keeping $E_{A}=E_{B}$, which gives in the small angle limit

$$
q_{z}=\mp p_{0} \Delta \theta, \quad q_{x}=q_{y}=0
$$

where $\theta_{A}=\theta_{B}=45^{\circ} \pm \Delta \theta$. In both these cases, the

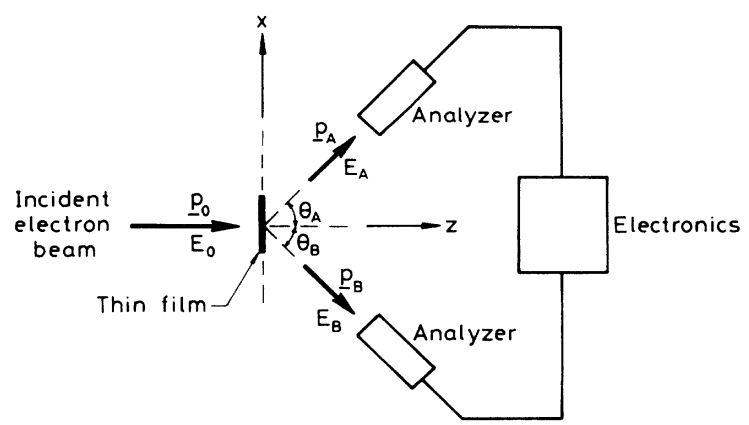

FIG. 1. Schematic of symmetric coplanar $(e, 2 e)$ scattering. Details of the electronics and data acquisition are found in Ref. 9. scattering angles were changed using parallel plate electrostatic deflectors. ${ }^{7}$

The major part of the data in this investigation was taken by varying the $x$ component of the momentum. This was done by keeping the three momentum vectors coplanar, letting $\theta_{A}=\theta_{B}=45^{\circ}$, and imposing an asymmetry in the energies of the two scattered electrons

$$
E_{A, B}=\frac{1}{2}\left(E_{0}+\epsilon\right) \pm \Delta E,
$$

giving

$$
q_{x}=\frac{\Delta E}{E_{0}}\left(1+\frac{\epsilon}{E_{0}}\right) p_{0}, \quad q_{y}=q_{z}=0
$$

where the variation in $q_{x}$ with binding energy is negligible $\left(<0.2 \%\right.$ for $\epsilon<20 \mathrm{eV}$ and $\left.E_{0}=10 \mathrm{keV}\right)$.

\section{B. Statistics}

The data collection time $T$ needed to obtain an accuracy $\delta \equiv \pm \Delta N_{t} / N_{t}$ (relative mean standard deviation) is given by (e.g., Lower and Weigold ${ }^{8}$ )

$$
T=\frac{1}{\delta^{2} \dot{N}_{t}}\left[1+\frac{1}{b}\left(1+\frac{1}{R}\right)\right],
$$

where $b$ is the signal-to-background ratio, $R$ is the ratio of background to coincidence timing window $(\approx 30$ in the present case), and $N_{t}$ and $\dot{N}_{t}$ are the true coincidence counts and count rate, respectively.

As an example, a statistical accuracy of $\pm 10 \%$ with a count rate per energy channel of $4 \mathrm{~min}^{-1}$ and $b=1$ requires an accumulation time of about $50 \mathrm{~min}$. A binding energy spectrum for a fixed momentum value may take two days, while a complete data set comprising ten different momentum values may take 20-30 days, depending on actual count rates recorded at higher momentum values. This situation sets high demands on the stability and mode of operation of the experiment. Long term drifts are compensated for by combining the results of multiple sweeps over the energy-momentum phase space of interest.

\section{EXPERIMENT}

\section{A. General}

The apparatus, ${ }^{9}$ comprising the electron gun, the sample manipulator, and two hemispherical analyzers, is enclosed in a stainless steel vacuum chamber equipped with turbomolecular and titanium sublimation pumps capable of maintaining a pressure of about $10^{-9}$ Torr. The magnetic field within the experimental volume is reduced to approximately $1 \mathrm{mG}$ by means of orthogonal pairs of Helmholtz coils.

The incident beam energy is $10 \mathrm{keV}$ and the incident current is $5 \mu \mathrm{A}$. The electron beam is generated by a commercial electron gun (Cliftronics Model $83 \mathrm{CE}$ ) and 
has an energy spread of $0.7 \mathrm{eV}$ from thermal plus spacecharge contributions. The beam is focused to a spot diameter of $0.5 \mathrm{~mm}$ on the film.

The coplanar geometry of the apparatus, in which the two scattered electrons are detected at angles $\theta=45^{\circ}$ relative to the incident beam, is shown in Fig. 1. The two detector subsystems are identical, each consisting of a solid-angle defining rectangular slit, a five-element retarding-focusing lens, a hemispherical energy analyzer, and dual microchannel plate detectors followed by a position sensitive resistive anode. The retarding-focusing lens reduces the energy of the scattered electron from 5 $\mathrm{keV}$ to $100 \mathrm{eV}$, the mean pass energy in the analyzer. The resolution of the analyzer is $1 \%$ of the mean pass energy. To a good approximation, the arrival position of an electron at the exit plane of the analyzer is proportional to its energy. Both the position information and a fast timing pulse are taken from the resistive anode. The energy resolution for the incident beam plus one detector was obtained from elastic scattering at $45^{\circ}$ of $5 \mathrm{keV}$ electrons from the carbon film and was $1 \mathrm{eV}$ [full width at half maximum (FWHM)]. The overall energy resolution for the coincidence measurements is estimated to be $1.5 \mathrm{eV}$ (FWHM). The momentum resolution of each analyzer, defined by the rectangular slit at the entrance of the detector, is 0.05 a.u. (FWHM), giving an overall momentum resolution for the coincidence spectrometer of 0.15 a.u. (FWHM).

Amorphous carbon films of thickness $80 \AA$ were purchased from ACF-METALS. The films were made by arc evaporation of carbon onto glass slides which were coated with a water soluble separation agent. They were floated off in distilled water and mounted across $2 \mathrm{~mm}$ diameter holes in an oxygen-free high-conductivity copper sample holder.

\section{B. Electronics and data acquisition}

The relative arrival times between electrons transmitted through the analyzers are measured by a time-toamplitude converter. Corrections are made to compensate for the energy-dependent time of flight variations occurring within the analyzers, thereby improving the time resolution of the experiment. The resulting timing spectrum in the inset of Fig. 2 shows a peak of "true" coincidence events resulting from pairs of electrons originating from unique $(e, 2 e)$ events, superimposed upon a flat background of "accidental events." Contributions from "accidental events" are easily subtracted due to their flat distribution in time. The signal $\left(\Delta t_{c}\right)$ and background $\left(\Delta t_{b}\right)$ windows as defined previously are indicated.

The energies of the two scattered electrons comprising each "true" coincidence event are determined through position-decoding electronics and then summed. The summed energy gives rise to a triangularly shaped response function centered at twice the mean pass energy of the analyzers with a full base $2 \Delta_{a}$, where $\Delta_{a}$ is the energy window of the analyzer. The "wings" of the response function are electronically truncated. The truncated response function is stepped incrementally over a selected

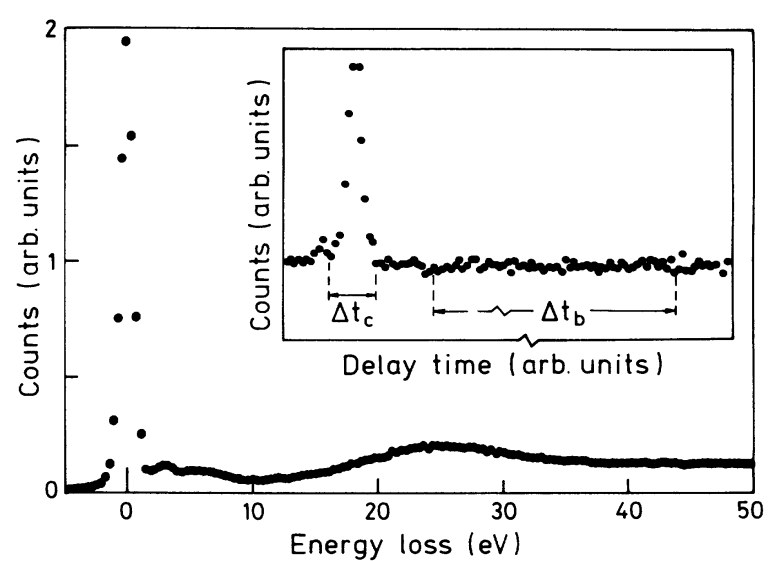

FIG. 2. The inset shows a typical coincidence timing spectrum. The enlarged dots fall within the signal window $\Delta t_{c}$. The main illustration shows scattering of $5 \mathrm{keV}$ electrons at an angle of $45^{\circ}$ as detected by one analyzer.

range of binding energies by stepping the incident energy. This allows one to cover a wider range of binding energy than $\Delta_{a}$ and also compensates for detector nonuniformities.

Each analyzer is calibrated by elastic scattering of 5 $\mathrm{keV}$ electrons at $45^{\circ}$, as shown in Fig. 2. The sharp peak at zero energy loss is due to elastic scattering and exhibits the instrumental energy resolution of $1 \mathrm{eV}$. In addition to the elastic peak, there is a broad energy loss maximum centered about $25 \mathrm{eV}$ due to double scattering events consisting of the elastic collision plus creation of a volume plasmon. Multiple scattering events are present in the raw coincidence spectra and have been deconvolved from the data by the method of Jones and Ritter. ${ }^{10}$

\section{RESULTS}

An example of the true coincidence spectrum at $q=0$ is shown in Fig. 3. The abscissa displays the binding energy relative to the Fermi energy. The solid curve in

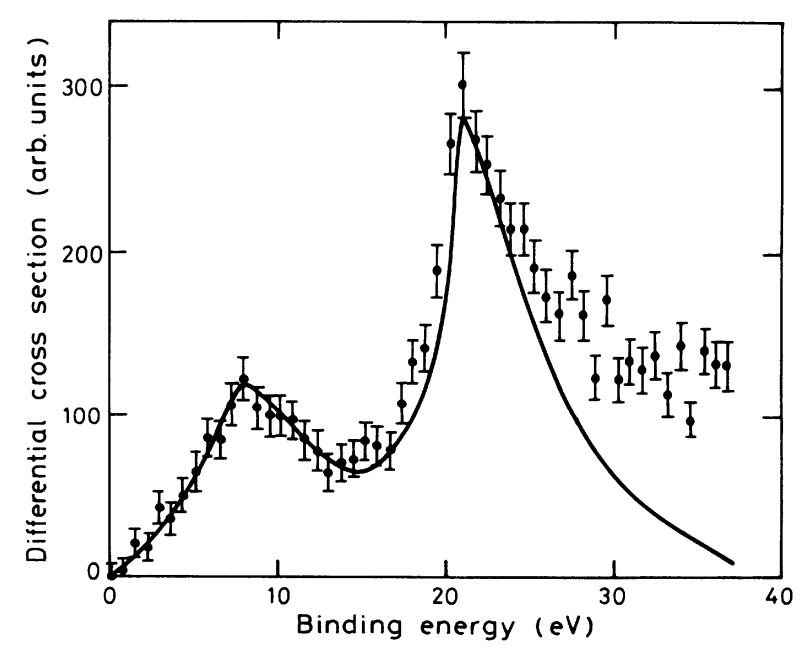

FIG. 3. Typical binding energy spectrum for $q=0$. 
Fig. 3 represents the deconvoluted data. The primary consequence of the deconvolution procedure is to remove the high binding energy tail which arises from multiple scattering. The calibration of the binding energy zero is confirmed by noting that the count rate goes to zero (within the one standard deviation error bars) at the Fermi energy. The distinct peaks at $8 \mathrm{eV}$ and $21 \mathrm{eV}$ will be discussed next.

The complete set of deconvoluted and smoothed data, i.e., coincidence rate as a function of binding energy for different momenta, is shown in Fig. 4(a). Two peaks are seen at low momentum. The peak at higher binding energy disperses upward to lower energy with increasing momentum while the peak at lower binding energy does not disperse. The same behavior has been observed in earlier $(e, 2 e)$ studies of amorphous graphitic carbon by Ritter et al. ${ }^{4}$ (energy resolution $6 \mathrm{eV} \mathrm{FWHM)} \mathrm{and} \mathrm{Hayes}$ et $a l .^{3}$ (energy resolution $4.5 \mathrm{eV}$ FWHM). In these two studies, the peak width was comparable to the energy resolution and, therefore, it was not possible to determine the intrinsic width of the peaks. In this study, the peak widths $(\geq 5 \mathrm{eV})$ are significantly greater than the energy resolution (1.5 eV FWHM) and are, therefore, quite close to the intrinsic width of these features.

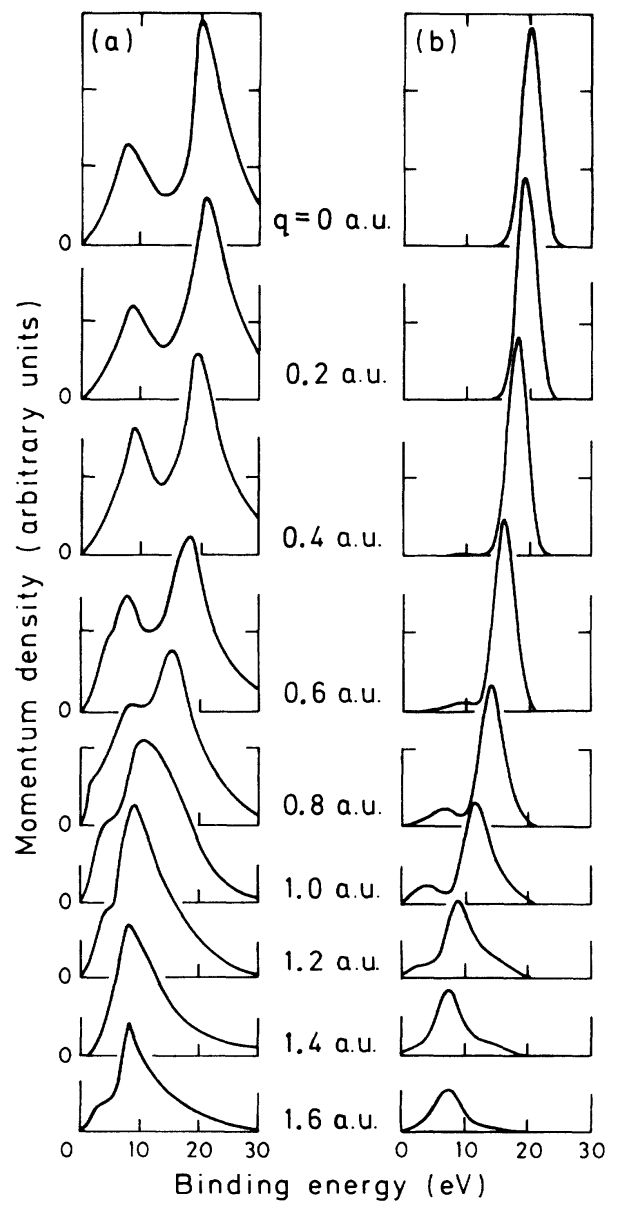

FIG. 4. Binding energy spectra for different momenta. (a) Experiment corrected for multiple scattering and excitation of plasmons, (b) numerical simulation by the spherically averaged spectral momentum density of the crystalline graphite.
The binding energies of the two peaks at zero momentum are equal within our error bars to the centroid energies of the two $\sigma_{1}$ and two $\pi$ bands of graphite at the Brillouin zone center $(21 \mathrm{eV}$ and $8 \mathrm{eV}$, respectively). For this reason, these two features in amorphous carbon have been identified with the corresponding features in graphite and have been labeled as $\sigma$ and $\pi$ bands. There are significant problems with attempting to make such a correspondence and they will be discussed in the next section. For purposes of discussion, we will refer to these two features as peak 1 and peak 2 for higher and lower binding energy peaks, respectively.

At higher momentum $q \geq 0.6$ an additional weak feature appears on the spectra as a shoulder at lower binding energy. The intensities of the features displayed in Fig. 4(a) were analyzed using commercial Gaussian curve fitting software. ${ }^{11}$ The integral intensities and positions of the peaks giving the best fit to the spectra are plotted in Figs. 5 and 6 , respectively.

Peak 1 decreases gradually with increasing momentum while peak 2 has an approximately constant intensity throughout the whole range of momenta. Peak 3 is an order of magnitude smaller than peaks 1 and 2 and its intensity can only be estimated to within $\pm 50 \%$ for $0.6 \leq q \leq 1.6$ a.u. (peak 3 was not resolved at 1.4 a.u.).

The behavior of peak 1 is associated with $s$-wave angular momentum symmetry while the behavior of the peak 3 is consistent with $p$-wave symmetry with intensity going through a maximum at $q \simeq 1$ a.u. We discuss this identification in Sec. $\mathrm{V}$ in more detail.

The appearance of features in the spectral momentum density of amorphous carbon which are similar to the energy bands of graphite naturally raises the question of whether our 80 - $\AA$-thick film is amorphous. Grill et al. ${ }^{12}$ for example, found that carbon bonding in plasma deposited, hydrogenated amorphous carbon films varied as a function of distance from the substrate over a dis-

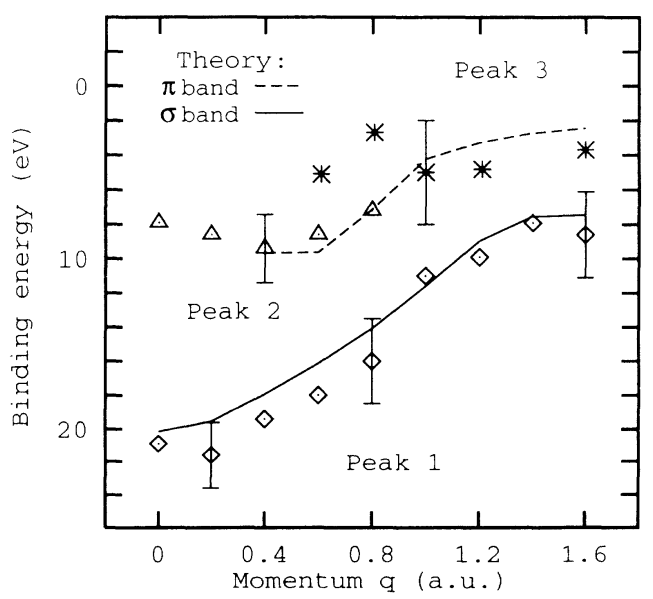

FIG. 5. Dispersion with momentum of the three peaks shown in Fig. 4. The diamonds are from the higher binding energy peak, the triangles are from the lower binding energy peak, and the asterisks are from the third minor peak seen as a shoulder on the spectra at $q \geq 0.6$. The solid and broken lines are the LMTO calculation for the $\sigma$ and $\pi$ bands, respectively. 


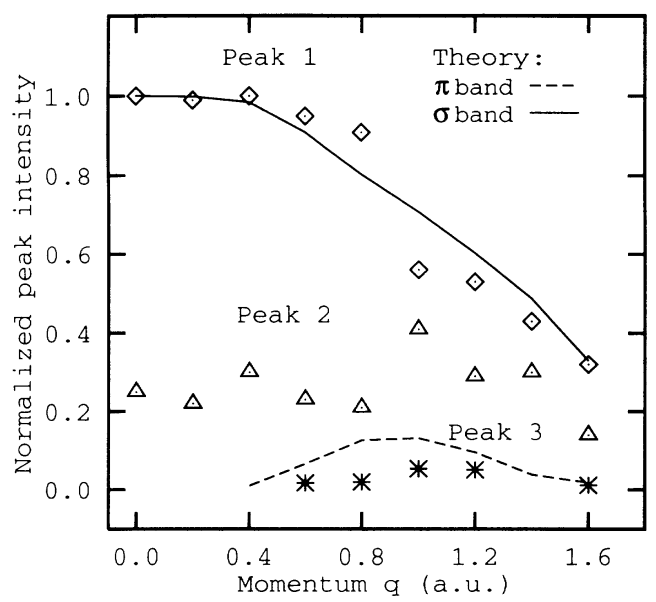

FIG. 6. Intensity as a function of momentum for the three peaks shown in Fig. 4(a). Legend as in Fig. 5.

tance of $400 \AA$. One could imagine that a graphitic amorphous carbon film might show preferential alignment of the $s p^{2}$ bonded sheets with the substrate. If this occurred, the spectral momentum density would be quite different if the momentum dependence were measured in the putative graphite basal plane, q parallel to the substrate, versus if it were measured perpendicular to the basal plane. For graphite, the binding energy of the band varies by over $5 \mathrm{eV}$ between $\mathrm{q}$ in the basal plane versus q perpendicular to the basal plane for $q=1.0$ a.u. ${ }^{13} \mathrm{We}$ have performed measurements with target electron momenta parallel and perpendicular to the film ( $x$ and $z$ directions, respectively) for $q=1.0$ a.u. The results are shown in Fig. 7. Within our error bars, the two measurements are identical. There is no shift of the peak at $10 \mathrm{eV}$ for the two momenta directions, indicating that

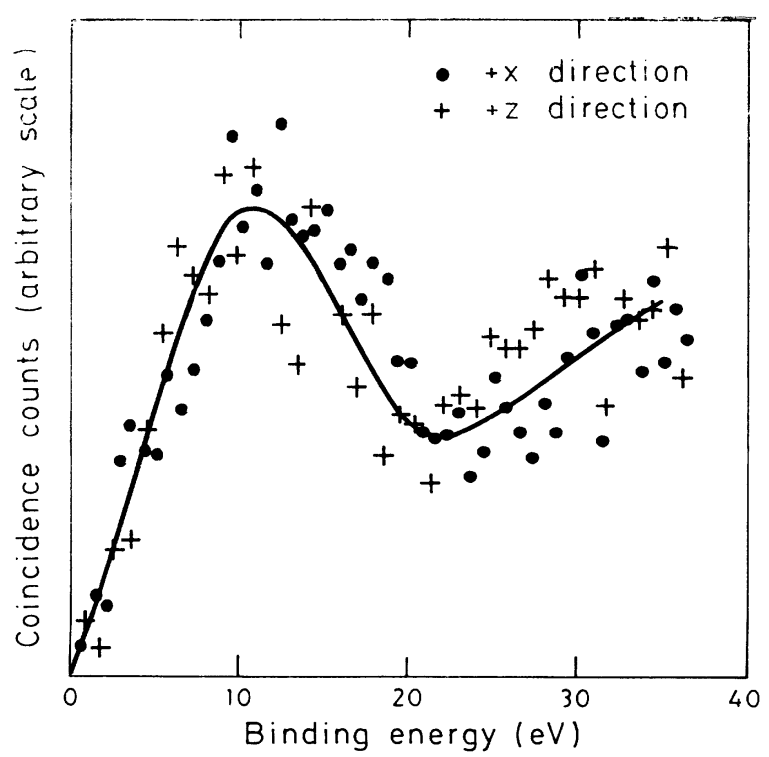

FIG. 7. Binding energy spectrum for magnitude of $q=1.0$ a.u. The crosses are data obtained for $\mathbf{q}$ in the $+z$ direction (see Fig. 1) and the solid circles are for data taken in the $+x$ direction. there is no preferential alignment of $s p^{2}$ bonded sheets in this film. Measurements in the two directions at other momenta support this conclusion. In addition, we have looked at films made by the same company with TEM and observed in diffraction only diffuse rings providing further evidence that the films are amorphous.

We now discuss a model of the spectral momentum density of graphitic amorphous carbon based on an angular average of the spectral momentum density of graphite.

\section{MODEL AND DISCUSSION}

\section{A. Model of amorphous graphitic carbon}

As alluded to in the last section, the dispersion with momentum of the features in the spectra shown in Fig. $4(a)$ is suggestive of graphite energy bands. There is, of course, a deep problem that energy bands, in the sense of crystalline $E$ versus $\mathbf{k}$, do not exist in amorphous solids because translational symmetry is absent. Some first-principles calculations of the spectral momentum density of amorphous, tetrahedrally bonded semiconductors do show vestiges of energy-momentum correlations in the amorphous form of the material which are similiar to the crystalline band structure. ${ }^{14-17}$ These calculations are based on highly simplified models of amorphous solids and are not of much help in understanding amorphous carbon with its multiple forms of possible local carbon bonding. To provide some insight into the electronic structure of this material, we introduce a heuristic model for the spectral momentum density of amorphous graphitic carbon based on an angular average of the graphite spectral momentum density. Though such an angular average of the crystalline structure is not consistent with diffraction measurements, it may provide a basis for understanding the electronic structure.

First, we calculate the band structure of graphite using the linear muffin-tin orbital (LMTO) method. ${ }^{18}$ This is an $a b$ initio self-consistent method which has an accuracy and computational efficiency sufficient to perform fullscale band structure calculations on solids with a large number of valence electrons per unit cell (16 in the case of graphite).

Some details of the calculated band structure of graphite are presented in Table I, where they are compared with the latest and most accurate pseudopotential calculation of Charlier et al. ${ }^{19}$ and experimental data on angular resolved photoemission spectroscopy of Eberhardt $e t a l .{ }^{20}$ and Law et al. ${ }^{21}$ Our data prove to be consistent both with the earlier calculation and the experiment, thus indicating applicability of the LMTO method to graphite.

Second, we calculate the spectral momentum density of the individual bands of graphite from the expression ${ }^{22}$

$$
\begin{aligned}
\rho_{j}(\epsilon, \mathbf{q})= & (2 \pi)^{-3} \sum_{\mathbf{G}} n_{j \mathbf{k}}\left|\int d^{3} r \psi_{j \mathbf{k}}(\mathbf{r}) e^{-i \mathbf{q} \cdot \mathbf{r}}\right|^{2} \\
& \times \delta\left(\epsilon-E_{j \mathbf{k}}\right) \delta_{\mathbf{q}, \mathbf{k}+\mathbf{G} .}
\end{aligned}
$$

Here $\psi_{j \mathbf{k}}$ and $E_{j \mathbf{k}}$ are, respectively, the one-electron wave 
TABLE I. Characteristic band energies (in eV) of graphite evaluated from the Fermi level.

\begin{tabular}{|c|c|c|c|c|}
\hline & \multicolumn{2}{|c|}{ Theoretical } & \multicolumn{2}{|c|}{ Experimental } \\
\hline & Present work & Charlier et $a l^{\mathrm{a}}$ & Eberhardt et al. ${ }^{\mathrm{b}}$ & Law et al. ${ }^{\mathrm{c}}$ \\
\hline \multirow[t]{2}{*}{ Bottom $\sigma$} & -20.1 & -20.1 & -20.6 & \\
\hline & -17.9 & -19.8 & & \\
\hline \multirow[t]{2}{*}{ Bottom $\pi$} & -11.9 & -8.9 & -8.1 & -8.5 \\
\hline & -7.6 & -6.8 & -7.2 & -6.6 \\
\hline \multirow[t]{2}{*}{ Top $\sigma$} & -5.1 & -3.5 & -4.6 & -5.5 \\
\hline & -5.1 & -3.4 & & \\
\hline
\end{tabular}

${ }^{\text {a }}$ Charlier et al. (Ref. 19).

${ }^{\mathrm{b}}$ W. Eberhardt et al. (Ref. 20).

${ }^{\mathrm{c}}$ A. R. Law et al. (Ref. 21).

function and energy defined by the band index $j$ and the crystal wave vector $\mathbf{k}, n_{\mathbf{j k}}$ is the occupation number of the corresponding one-electron state. The integration in Eq. (8) is carried over the unit cell where the wave function $\psi_{j \mathbf{k}}$ is normalized to unity. The reciprocal lattice vector $\mathbf{G}$ translates the momentum $\mathbf{q}$ to the first Brillouin zone. The spectral momentum density is normalized over energy and momentum space to the number of valence electrons per unit cell per spin:

$$
2 \sum_{j} \int d \epsilon d^{3} q \rho_{j}(\epsilon, \mathbf{q})=N_{e} .
$$

In the LMTO method the wave function is expanded over spherical harmonics

$$
\psi_{j \mathbf{k}}(\mathbf{r})=\sum_{l m} a_{l m}^{j \mathbf{k}} i^{l} Y_{l m}(\hat{r}) \frac{1}{r} P_{k l}(r)
$$

where $a_{l m}^{j \mathbf{k}}$ are the expansion coefficients for the muffintin orbitals and $P_{k l}(r)$ are the radial orbitals normalized to unity over the muffin-tin sphere. Substituting this expansion into Eq. (8) gives

$$
\begin{aligned}
\rho_{j}(\epsilon, \mathbf{q})= & \frac{2}{\pi} \sum_{\mathbf{G}} n_{j \mathbf{k}}\left|\sum_{l m} a_{l m}^{j \mathbf{k}} Y_{l m}(\hat{r}) \int_{0}^{S} d r j_{l}(q r) P_{k l}(r)\right|^{2} \\
& \times \delta\left(\epsilon-E_{j \mathbf{k}}\right) \delta_{\mathbf{q}, \mathbf{k}+\mathbf{G}}
\end{aligned}
$$

where $s$ is the muffin-tin radius and $j_{l}(\mathrm{qr})$ is the spherical Bessel function. Equation (11) is readily generalized to the case of several nonequivalent atoms per unit cell by the additional summation over the number of muffin tins with the phase factor $\exp \left(-i \mathbf{q} \cdot \mathbf{R}_{i}\right)$ in which $\mathbf{R}_{i}$ is the coordinate of the center of the corresponding muffin tin.

In Fig. 8 we present the band structure and momentum density of graphite calculated along the basis vectors of the reciprocal lattice. An analogous calculation was reported earlier by Gao et al. ${ }^{23}$ Their results are in a fair agreement with ours. The characteristic feature of the band structure is the doubled valence bands $\pi, \sigma_{1}$, $\sigma_{2}$, and $\sigma_{3}$. These doubled bands are the consequence of the layered structure of graphite with the interlayer
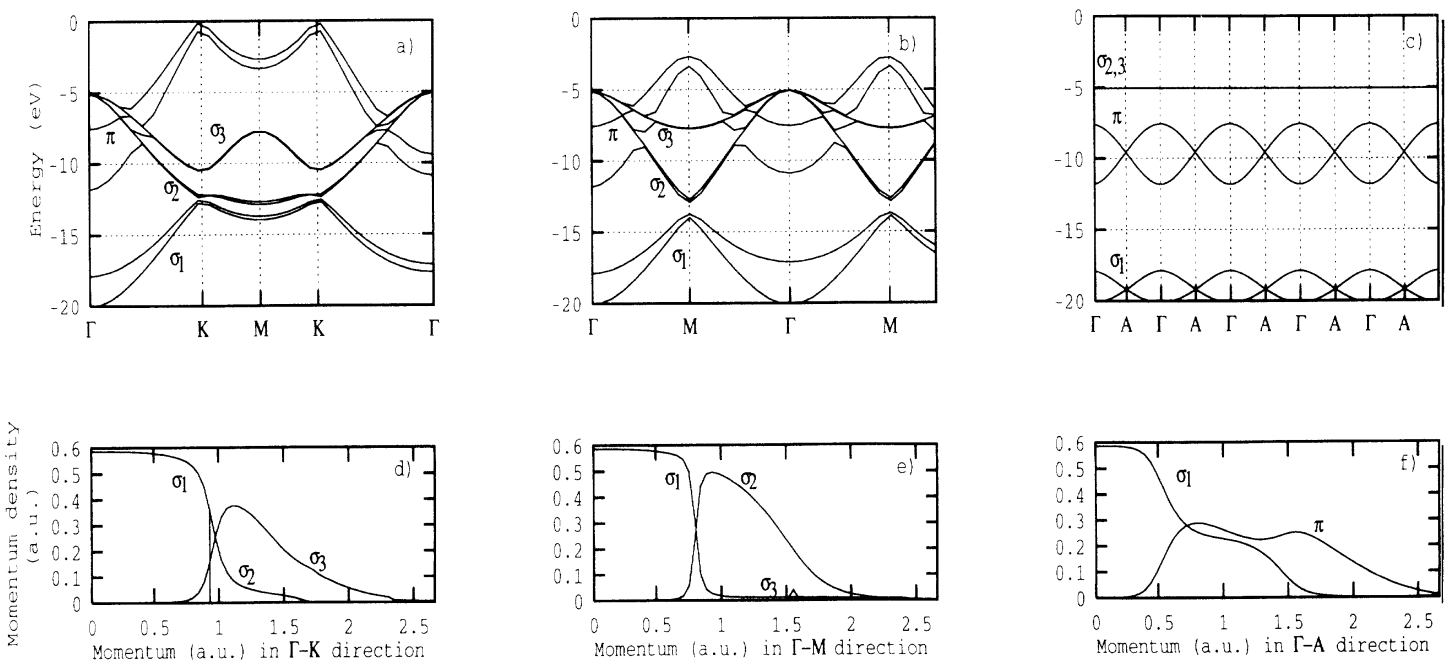

FIG. 8. Energy bands (a)-(c) and momentum densities (d)-(f) of graphite in several directions in $\mathbf{k}$ space. 
separation much larger than the distance between neighboring atoms in the layer. The momentum densities of the $\pi$-band orbitals are zero for $q$ in the $\Gamma-K-M$ plane, since they have a node there. Similarly, for symmetry reasons, ${ }^{24}$ the momentum densities of the $\sigma_{2^{-}}$and $\sigma_{3^{-}}$ band orbitals are zero along the $c$ axis. In an arbitrary direction out of the basal plane all four bands have nonvanishing momentum densities. The momentum densities of the $\sigma_{1}$ orbitals fall off monotonically with $q$ consonant with their $s$-wave symmetry. Note that in the extended zone scheme, the $\sigma_{1}$-band momentum densities meld continuously with the $\sigma_{2}$-band densities along the $\Gamma-M$ direction following the nearly free electron parabola.

Finally, we take an angular average of Eq. (11) to obtain our model for the spectral momentum density of amorphous carbon

$$
\begin{aligned}
\rho(\epsilon, q) & =\sum_{j}(4 \pi)^{-1} \int d \Omega_{\mathbf{q}} \rho_{j}(\epsilon, \mathbf{q}) \\
& =\sum_{j} \frac{6}{\pi} \int_{0}^{\pi / 2} \cos \vartheta_{q} d \vartheta_{q} \int_{0}^{\pi / 6} d \varphi_{q} \rho_{j}(\epsilon, \mathbf{q}),
\end{aligned}
$$

where the angular averaging is carried out over the irreducible wedge of the Brillouin zone of the hcp crystal structure. In order to simulate the finite energy resolution of the experiment, we substitute the delta function $\delta\left(\epsilon-E_{j \mathbf{k}}\right)$ in Eq. (11) with a Gaussian of the appropriate width. The final results are shown in Fig. 4(b) where the spherically averaged momentum densities are plotted as functions of the binding energy for different momenta. The same momenta are implicit variables in Figs. 4(a) and 4 (b) so that experiment and calculation can be compared directly. The peak positions and intensities for the separate $\sigma$ and $\pi$ bands [restricting the summation over the band index $j$ in Eq. (11)] are plotted in Figs. 5 and 6 as solid and dashed lines, respectively.

\section{B. Discussion}

Some features of our model are in qualitative agreement with the experimental measurements while other features are in clear disagreement. Consider first where the model provides some basis for understanding the spectral momentum density of amorphous graphitic carbon.

The dispersion of the first peak in the data [Fig. 4(a)] with momentum is simulated well by the peak [Fig. 4(b)] in the angularly averaged spectral momentum densities associated with the three $\sigma$-bands in graphite. The strong parabolic dispersion of the model peak may be unexpected given the weak dispersion of the $\sigma$ bands for momentum along the $c$ axis. But the angular average is heavily dominated by momentum in the basal plane, where the dispersion is parabolic, since the equatorial angular phase space is so much greater than the polar phase space. Similarly, the intensity of the first peak in the data agrees well with our model where again the angular average is heavily dominated by contributions from momentum in the basal plane (roughly an average of the $\Gamma-K$ and $\Gamma-M$ directions).
The major qualitative difference between the data and model is in the low binding energy region between the Fermi energy and $\sim 12 \mathrm{eV}$. The data display in this energy range a strong peak (peak 2 in Figs. 5 and 6) with significant intensity at low momenta plus a weak shoulder (peak 3 in Figs. 5 and 6) in the higher momenta spectra $q \geq 0.6$ a.u. Besides the first peak discussed in the preceding paragraph, our model displays only one other weak feature associated with the angular average of the graphite $\pi$-band spectral momentum density. The model $\pi$-band dispersion (dashed line in Fig. 5 ) is dominated by contributions from the angular average which lie between the equatorial plane and the poles. The model $\pi$-band intensity (dashed line, Fig. 6) goes to zero at small momenta and the peak in the intensity occurs at $q \simeq 1.0 \mathrm{a} . \mathrm{u}$. Peak 2 and peak 3 in the data are dispersionless within our error bars and demarcate the upper and lower bounds of the model $\pi$-band dispersion. The intensity of peak 3 has a large uncertainty from the fitting procedure, but it may have the same momentum dependence as the model $\pi$-band intensity. Clearly, one cannot identify either peak 2 or peak 3 with our model.

The most puzzling feature of the experimental spectra at low binding energy is peak 2. The energy of this peak is almost exactly the same as the zone center graphite $\pi$ band, but the momentum densities of the $\pi$-band states in graphite go to zero at the zone center, whereas peak 2 is quite intense in the zero momentum limit. In a study of diamondlike amorphous carbon by $(e, 2 e)$ spectroscopy ${ }^{25}$ two peaks were observed in the spectral momentum density which were qualitatively similar to peak 1 and peak 2 of our data. The resolution was insufficient to resolve peak 3 if it existed. Again, a peak in the diamondlike amorphous carbon spectra at the zone center energy of the graphite $\pi$ band had significant intensity at zero momentum. It was suggested that this behavior of the low binding energy feature might be due to rehybridization of graphitic $\pi$ and $\sigma$ orbitals which would admix $s$-wave symmetry character into the $\pi$ orbital (explaining the finite intensity of the momentum density at zero momemtum). ${ }^{26}$ The rehybridization may be due either to bending and wrinkling of $s p^{2}$ bonded sheets or, as argued by Tamor and $\mathrm{Wu},{ }^{27}$ to puckering of the sheets where there is intersheet crosslinking.

If there is significant rehybridization of the $\pi$ orbitals going from graphite to amorphous carbon, one would expect a similar mixing of $s$-wave symmetry into the $p$-like states of the graphitic $\sigma_{2}$ and $\sigma_{3}$ bands. The two peaks in our data which fall in the same energy range as the graphitic $\pi-, \sigma_{2^{-}}$, and $\sigma_{3}$-bands might be rehybridized counterparts of the crystal states. The different intensities of peak 2 and peak 3 in the data might arise from the different degree of $s$-wave admixture for $p$-like orbitals which are perpendicular to the $s p^{2}$ bonded sheets compared to $p$-like orbitals lying in the $s p^{2}$ bonded sheets. Resolution of this question will require a more sophisticated model of amorphous carbon than ours.

To check whether the spectral momentum we have measured by $(e, 2 e)$ spectroscopy is reasonable, we numerically integrate the experimental spectra $N_{c}(\epsilon, q)$ shown in Fig. 4(a) over momenta to obtain the density 


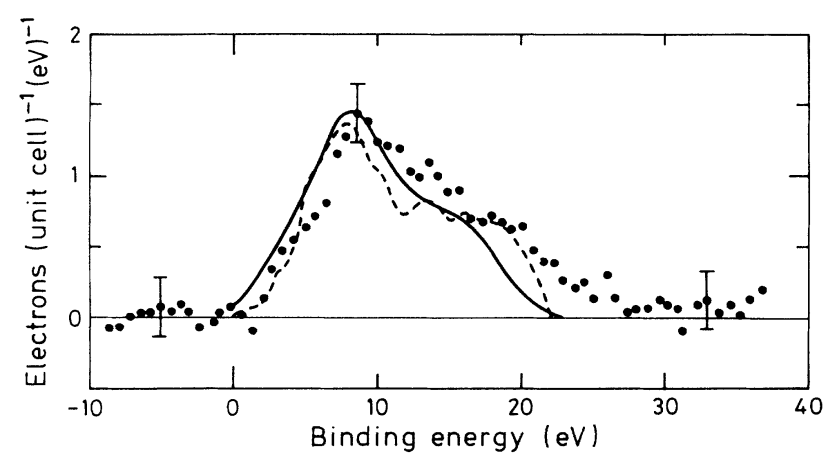

FIG. 9. The points with representative error bars represent $\sum_{q_{i}} q_{i}^{2} N_{c}\left(\epsilon, q_{i}\right)$. The solid curve is based on the present theory convoluted with an energy resolution of $1.5 \mathrm{eV}$. The broken line is from the photoemission experiment of Ref. 28 on crystalline graphite.

of states

$$
D(\epsilon)=\int 4 \pi \rho(\epsilon, q) q^{2} d q \rightarrow \sum_{q_{i}} q_{i}^{2} N_{c}\left(\epsilon, q_{i}\right)
$$

The results of this analysis are the solid circles shown in Fig. 9. The high energy tail in the data is probably due to incomplete deconvolution of the multiple scattering contributions. The solid line in the figure is the density of states obtained from our model. The dashed line is the photoemission measurement of Bianconi et al. ${ }^{28}$ on crystalline graphite which is quite similar to photoemission measurements on annealed samples of hydrogenated amorphous carbon. ${ }^{29}$ The agreement between the density of states determined from the $(e, 2 e)$ spectra and the density of states measured by photoemission spectroscopy corroborates our analysis of the $(e, 2 e)$ data to obtain the spectral momentum density.

\section{CONCLUSIONS}

We have presented measurements of the spectral momentum density of arc evaporated amorphous carbon by $(e, 2 e)$ spectroscopy with significantly improved energy resolution and data rate. Our measurements have been compared to a heuristic model of amorphous carbon based on an angular average of the spectral momentum density of crystalline graphite. An intense feature in our data which disperses parabolically from a binding energy of $21 \mathrm{eV}$ at zero momentum to approximately $8 \mathrm{eV}$ at higher momentum ( $q \simeq 1.4$ a.u.) is described well by our model. A weaker, dispersionless feature at a binding energy of $\simeq 8 \mathrm{eV}$ and a dispersionless shoulder at a binding energy of $\simeq 4 \mathrm{eV}$ cannot be explained by our model. We suggest that these two low binding energy features in the data might be associated with rehybridization of the graphitic $\pi$ orbitals and the two graphitic $p$-wave-like $\sigma$ orbitals.
* Present address: Research School of Physical Sciences and Engineering, Australian National University, Canberra, ACT 2601.

${ }^{1}$ J. C. Angus and C. C. Hayman, Science 241, 913 (1988).

2 J. Robertson, Prog. Solid State Chem. 21, 199 (1991).

${ }^{3}$ P. Hayes, J. F. Williams, and J. Flexman, Phys. Rev. B 43, 1928 (1991).

${ }^{4}$ A. L. Ritter, J. R. Dennison, and R. Jones, Phys. Rev. Lett. 53, 2054 (1984).

${ }^{5}$ I. E. McCarthy and E. Weigold, Rep. Prog. Phys. 51, 299 (1988).

${ }^{6}$ J. F. Williams and P. A. Hayes, Aust. J. Phys. 43, 465 (1990).

7 A. L. Ritter, J. R. Dennison, and J. Dunn, Rev. Sci. Instrum. 55, 1280 (1984).

${ }^{8} \mathrm{~J}$. Lower and E. Weigold, J. Phys. E 22, 421 (1989).

${ }^{9}$ J. Lower, S. M. Bharathi, C. Yu, K. J. Nygaard, and E. Weigold, Surf. Sci. 251/252, 213 (1991).

${ }^{10}$ R. Jones and A. L. Ritter, J. Electron Spectrosc. Relat. Phenom. 40, 285 (1986).

${ }^{11}$ GRAMS $/ 386^{\mathrm{TM}}$ User's Guide, Galactic Industries Corporation, 1991-1992.

${ }^{12}$ A. Grill, B. S. Meyerson, V. V. Patel, J. A. Reimer, and M. A. Petrich, J. Appl. Phys. 61, 2874 (1987).

${ }^{13}$ N. A. W. Holzwarth, S. G. Louie, and Sohrab Rabii, Phys. Rev. B 26, 5382 (1982).

${ }^{14}$ D. P. DiVincenzo, R. Mosseri, M. H. Brodsky, and J. F.
Sadoc, Phys. Rev. B 29, 5934 (1984).

${ }^{15}$ J. L. Beeby and T. M. Hayes, Phys. Rev. B 32, 6464 (1985).

${ }^{16}$ R. Haydock, J. Non-Cryst. Solids 75, 121 (1985).

${ }^{17}$ B. J. Hickey and G. J. Morgan, J. Phys. C 19, 6195 (1986).

${ }^{18}$ H. L. Skriver, The LMTO Method (Springer, New York, 1984).

19 J.-C. Charlier, X. Gonze, and J.-P. Michenaud, Phys. Rev. B 43, 4579 (1991).

${ }^{20}$ W. Eberhardt, J. T. McGovern, E. W. Plummer, and J. E. Fischer, Phys. Rev. Lett. 44, 200 (1990).

${ }^{21}$ A. R. Law, J. J. Barry, and H. P. Hughes, Phys. Rev. B 28, 5332 (1983)

${ }^{22}$ B. I. Lundqvist and C. Lydén, Phys. Rev. B 4, 3360 (1971).

${ }^{23}$ Chao Gao, A. L. Ritter, J. R. Dennison, and N. A. W. Holzwarth, Phys. Rev. B 37, 3914 (1988).

${ }^{24}$ R. Harthoorn and P. E. Mijnarends, J. Phys. F 8, 1147 (1978).

${ }^{25}$ Chao Gao, Yun Yu Wang, A. L. Ritter, and J. R. Dennison, Phys. Rev. Lett. 62, 945 (1989).

${ }^{26}$ R. C. Haddon, J. Am. Chem. Soc. 108, 2837 (1986).

${ }^{27}$ M. A. Tamor and C. H. Wu, J. Appl. Phys. 67, 1007 (1990).

${ }^{28}$ A. Bianconi, S. B. M. Hagström, and R. Z. Bachrach, Phys. Rev. B 16, 5543 (1977).

${ }^{29}$ D. Wesner, S. Krummacher, R. Car, T. K. Sham, M. Strongin, W. Eberhardt, S. L. Weng, G. Williams, M. Howells, F. Kampas, S. Heald, and F. W. Smith, Phys. Rev. B 28, 2152 (1983). 